\title{
EMPRENDIMIENTO COMO PROCESO DE RESPONSABILIDAD SOCIAL EN INSTITUCIONES DE EDUCACIÓN SUPERIOR EN COLOMBIA Y VENEZUELA ${ }^{1}$
}

\section{THE ENTREPRENEURSHIP AS A PROCESS OF SOCIAL RESPONSIBILITY IN INSTITUTIONS OF HIGHER EDUCATION IN COLOMBIA AND VENEZUELA}

\author{
Duran Sonia ${ }^{2}$; Fuenmayor Alejandro ${ }^{3}$; Cárdenas Sergio ${ }^{4} \&$ Hernández Rene $^{5}$ \\ Corporación Universitaria Latinoamericana / Universidad Dr. Rafael Belloso Chacín. Zulia-Venezuela / \\ Universidad del Zulia-Venezuela
}

RECIBIDO: Febrero 18 de 2016

ACEPTADO: Junio 13 de 2016

\section{RESUMEN}

El estudio está orientado a caracterizar el emprendimiento como proceso de responsabilidad social en instituciones de educación superior en Colombia y Venezuela, para alcanzar objetivos rentables que contribuyan a la generación de bienestar y crecimiento social. La Investigación fue de tipo descriptivo, no experimental, de campo, la población estuvo conformada por cuarenta (40) docentes especialistas en emprendimiento. La técnica de recolección de datos fueron dos cuestionarios para medir emprendimiento y responsabilidad social. Compuestos por (15) ítems cada uno, con diferentes alternativas tipo escala likert. La validez se obtuvo a través del juicio de expertos. Para el cálculo de la confiabilidad se utilizó el método de alfa Cronbach, donde se obtuvo un valor de 0.80 para cada variable, comprobando que el instrumento es confiable. Los datos obtenidos fueron interpretados estadísticamente mediante frecuencia absoluta y relativa, además agrupados por dimensión e indicador. Se concluye que contar con herramientas para el emprendimiento radica en la posibilidad de desarrollar políticas de desarrollo y de responsabilidad social en un contexto en que éste ha cambiado notablemente a partir del proceso educativo

Palabras clave: Emprendimiento; responsabilidad social; desarrollo económico; instituciones de educación superior. L26, M14, O1, O43.

\begin{abstract}
The study is oriented to characterize entrepreneurship as a process of social responsibility in higher education institutions in Colombia and Venezuela, in order to achieve profitable objectives that contribute to the generation of well-being and social growth. The research was descriptive, not experimental, field work; the population was made up of forty (40) teachers specializing in entrepreneurship. The technique of data collection was two questionnaires to measure entrepreneurship and social responsibility. Composed by (15) items each, with different likert scale type alternatives. Validity was obtained through expert judgment. For the calculation of reliability, the alpha Cronbach method was used, where a value of 0.80 was obtained for each variable, proving that the instrument is reliable. The obtained data were interpreted statistically by absolute and relative frequency, also grouped by dimension and indicator. As a conclusion, having tools for entrepreneurship lies in the possibility of developing development policies and social responsibility in a context in which it has changed significantly from the educational process.
\end{abstract}

Keywords: Entrepreneurship, social responsibility, economic development - Institutions and growth. L26, M14, O1, O43.

\section{Este artículo se puede referenciar}

Durán, S., Fuenmayor, A., Cárdenas, S. \& Hernández, R. (2016). Emprendimiento como proceso de responsabilidad social en instituciones de educación superior en Colombia y Venezuela. En Desarrollo Gerencial Revista de la Facultad de Ciencias Económicas Administrativas y Contables de la Universidad Simón Bolívar-Colombia, 8(2), 58 - 75.

\footnotetext{
I Investigación resultado del proyecto "La formación del emprendimiento un elemento en el contexto internacional"

2 Doctora en Ciencias Gerenciales, Docente - Investigadora Corporación Universitaria. Latinoamericana. Barranquilla Colombia, Soniaduran547@hotmail.com.

3 Doctor en Ciencias Gerenciales, Docente e Investigador de pre y post grado. Universidad Rafael Belloso Chacín, afuenmayor3x3@yahoo.com.ve.

${ }^{4}$ Candidato a Doctor en Ciencias Gerenciales Urbe Internacional, Docente de Posgrados en Finanzas y Revisoría Fiscal Universidad Javeriana, Unilibre Seccional Barranquilla. Docente de pregrado Corporación Universitaria Latinoamericana- Actual. sergiocardenast@hotmail.com

${ }^{5}$ Dra. En Ciencias Gerenciales, profesora titular de la Universidad del Zulia, Directora del Departamento de Ciencias Humanas núcleo Costa Oriental Correo: reneeher@gmail.com
}

Desarrollo Gerencial, 8 (2) Pp. 58 - 75 Julio-Diciembre 2016. ISSN: 2145-5147 (On Line). Universidad Simón Bolívar. Barranquilla-Colombia.Contactos: desarrollogerencial@unisimonbolivar.edu.co 


\section{INTRODUCCIÓN}

En la actualidad, las sociedades se encuentran inmersas en un proceso de cambio continuo, situación desembocada por una cantidad de situaciones irregulares que se han generado desde las comunidades y hacia ellas, planteando procesos para que la sociedad participe y haga aportes de tipo institucional, social y solidario con miras a mejorar la calidad de vida de dichas comunidades. Dentro de este marco, en la búsqueda de marcar la diferencia en la manera de crear cultura social y comunitaria, las instituciones de educación superior a nivel mundial se han encaminado a promover programas y procesos de desarrollo y apoyo a la sociedad.

Es así como se concibe en la actualidad el proceso de emprendimiento, el cual se ha enfocado al trabajo con las comunidades en condiciones de vulnerabilidad, pero ademas apoyar a las personas o grupos con la disposición de promover una idea de negocios, de tal manera de generar un proceso de integración como un sistema de direccionamiento de políticas de desarrollo social, económico y personal, promoviendo el desarrollo de las comunidades, orientándose al emprendimiento como base para cumplir con la responsabilidad social, esto significa emprender desde las necesidades de las comunidades y para contrarrestarlas. Dando paso a la vinculación entre la empresa y la universidad.

Para Castillo (2010), existe un modelo de la Triple Hélice, el cual plantea un nuevo paradigma que propicia una misión emprendedora para la universidad. A partir de este enfoque, la noción de universidad emprendedora se ha desarrollado con mucha más importancia, utilizando avances para el desarrollo socioeconómico, actividades de patentes, y licencias e institucionalización de actividades spin-off. Esto ha producido un cambio de actitud entre los académicos respecto a la colaboración con la empresa.

Bajo las circunstancias actuales de crisis mundial, Paz, Prieto y García (2016), consideran que emprender no es únicamente una oportunidad, sino una necesidad. Debido a su naturaleza creadora de riqueza, la acción de emprendimiento no solo tiene un sentido económico, generando productividad, sino que también posee una marcada connotación social, creando fuentes de empleo, con lo cual se incide en un crecimiento económico equitativo e incluyente que permite participar de los beneficios económicos a quienes menos ingresos tienen. Esta actividad emprendedora se ve reforzada en el caso de la universidad, pública y privada, por el papel relevante que se espera de ella como generadora de aptitudes y modelos a imitar a escala social.

Esta situación se ha ido concretando desde las necesidades socioeconómicas de los actores sociales, para que estos se adapten a los cambios culturales y sociales originados por el proceso de globalización, cabe destacar que las IES públicas y privadas han asumido el rol de responder a las necesidades sociales, preocupándose por estandarizar procesos de integración e inserción social, vislumbrando la necesidad de 
responder a los lineamientos de cooperación, calidad y preservación del entorno, desarrollando la generación de ingresos para su subsistencia y de esta manera cumplir con su responsabilidad social.

Desde estas perspectivas, un mercado competitivo y globalizado no solo se ve reflejado ante las organizaciones destinadas adquirir un interés económico sino que al igual requiere de la concepción e integración de organizaciones académicas por ser parte de ese mundo complejo que inunda la actualidad social, donde las funciones de tal son ejercidas a través de un liderazgo ético, con capacidades de velar no solo de su campo personal, sino institucional, para así garantizar la sustentabilidad y sostenibilidad de ellas en el sector académico, aunado al compromiso por forjar desde su rol la sociedad con principios éticos-morales que conlleve a un ejercicio profesional transparente en la realización de sus actividades diarias de gestión.

En este sentido la investigación tuvo como finalidad identificar las herramientas y analizar las tácticas ejecutadas para fomentar el emprendimiento desde las instituciones de educación superior, apoyando las ideas de negocios y la innovación de las comunidades educativas y sociales. En ese mismo ámbito se determina como se ha incursionado desde el emprendimiento hacia los negocios inclusivos verificando si este se concibe como parte de un proceso de responsabilidad social.

Dentro de este marco, Castillo (2010) sostiene que las investigaciones de las universidades deben ser planteadas desde una perspectiva de necesidades de cooperación entre las empresas, con el propósito de desarrollar nuevos productos y estrategias que ayuden a fortalecer y generar nuevas tecnologías, la diversificación de los nuevos conocimientos, el desarrollo, así como la investigación e innovación para compartir entre ambas. Como una estrategia de apoyo a la sociedad en general.

Bajo este contexto, para este proceso de emprendimiento se debe tomar en cuenta el modo de vida, las condiciones de vida y el nivel de vida, consideradas como categorías de corte sociológico que se relacionan estrechamente con el bienestar. Entre las condiciones de vida están, la disponibilidad de fuentes de trabajo, proceso que desde este estudio se ha buscado promover, fomentando la generación de ingresos, idea de negocios, a través de un proceso de emprendimiento orientado a un emprendimiento solidario y cultural, arraigado a la mejora de las condiciones de vivienda, servicios de atención médica, disponibilidad de alimentos, existencia de centros culturales, deportivos, saneamiento ambiental, transporte, comunicaciones, entre otros.

Para lograr lo antes planteado, se han buscado alternativas que coadyuven a estos grupos de interés a superar de alguna manera las limitaciones y problemas acaecidos como consecuencia de los problemas sociales y económicos, (vulnerabilidad). Lo que se pretende es generar ingresos, a partir del emprendimiento social, solidario y cultural, considerando al individuo como un ser bío-psico y social, de 
esta manera propiciar la integración de los ciudadanos desde los negocios inclusivos, comprendiéndolos desde diferentes perspectivas y realidades.

Uno de los aspectos que más relevancia para este contexto, en opinión de Duran, Parra y Márceles (2015) es la socialización emprendedora, concebida como un conjunto de experiencias relacionadas con la realización exitosa de las tareas emprendedoras, que pueden ser fácilmente establecidas a partir de la interacción con personas que poseen características emprendedoras que sirven como modelos a imitar.

En tal sentido, existe la necesidad de desarrollar estrategias para promover con efectividad y calidad en el ambiente donde se desenvuelven, para lo cual el ciudadano representa uno de los factores más importante, sumado esto a los cambios estratégicos para promover el desarrollo económico, la generación de ingresos y el emprendimiento, los cuales deben estar apoyados por los valores, actitudes y conducta de su gente, ello permite fomentar la responsabilidad social de las IES, promoviendo los negocios inclusivos para alcanzar los objetivos de las comunidades.

En opinión de Perdomo y Prieto (2009) estas instituciones requieren del apoyo de un liderazgo con capacidades en la asunción de retos centrados en valores y con sentido de identidad, apuntando así en su representación social hacia la responsabilidad ética; desde luego que destacando actividades emprendedores sustentadas en la autonomía, flexibilidad y comprometidas con la filosofía de gestión organizacional. Es decir, se trata de consolidar la misión de formación del ser humano desde una proyección objetiva, mediante capacidades valorativas e tanto ente moral y ético

El mismo implica realizar un conjunto de estrategias y procesos para lograr dirigir la aplicación de los mismos, ayudando en el proceso de generación de ingreso sustentado en el emprendimiento social así como en la convivencia ciudadana, de tal manera de lograr sus objetivos y metas propuestas. Dentro de esta perspectiva, se ubica la responsabilidad social, lo cual es concebido por Certo (2007), como la actitud voluntaria de la empresa dirigida a satisfacer las demandas sociales de los distintos agentes relacionados con la misma, derivados de los efectos que sobre los mismos provoca la actividad empresarial. Se sugiere que cada empresa, en función de sus valores y compromisos sociales, debe definir el campo de actuación de su responsabilidad social.

Atendiendo a estas consideraciones las organizaciones, deben asumir cierto grado de responsabilidad social, incorporando a la planificación objetivos socio-económicos vinculados a los grupos que intervienen directa o indirectamente en su desarrollo, intentando buscar un equilibrio entre las funciones económicas y sociales. En tal sentido, Duran \& otros (2016) coinciden en cualquier acción llevada a cabo por una empresa afecta al bienestar de sus grupos de interés. Por ende una determinada estrategia puede aumentar el bienestar de algunos y causar daño a otros. 
Por tanto, la responsabilidad social para Prieto \& Paz (2011), ha de incorporarse a la estrategia de las IES, debe estar íntimamente ligada a su proceso de planificación estratégica y a su correspondiente control, ella se asocia con las relaciones, obligaciones y deberes generalmente aceptados que las universidades objeto de estudio tienen con la sociedad, involucrando la integración voluntaria, por parte de las empresas, de las preocupaciones sociales y medioambientales en sus operaciones comerciales y en las relaciones con sus interlocutores. En este orden de ideas, las organizaciones se muestran partidarias de dedicar esfuerzos a programas orientados a mejorar su entorno físico - social, promoviendo el crecimiento y desarrollo de las comunidades externas, ello influye positivamente en la opinión pública, en pro de incrementar su capacidad de atención e indirectamente los beneficios a largo plazo, sin costes añadidos.

Con respecto a la importancia del estudio, este tiene alto grado de pertinencia social, dado que busca analizar las características del emprendedor en función del desarrollo de habilidades de autogestión y autonomía, esto sustentado en Duran, Parra, Martínez, Hernández \& Otros (2016)para promover el emprendimiento, el mismo está orientado a ofrecer herramientas que incrementen los índices de emprendimiento e ideas de negocios, sustentado en procesos de investigación, desarrollo e innovación, desde las instituciones de educación superior como proceso de su responsabilidad social.

En tal sentido se determina si las IES como parte de su responsabilidad social se vincula con el ciudadano y su comunidad desde su rol protagónico y participativo. Por otro lado, pretende ampliar el abanico de posibilidades con las que cuentan los ciudadanos para su desarrollo emprendedor además de la vinculación con su entorno social. En función de generar los procesos de emprendimiento desde diferentes perspectivas, tales como emprendimiento económico, Comunitario, Emprendimiento Solidario y Emprendimiento Cultural. En este sentido, las IES están orientada a generar beneficios, los mismos contribuyen al logro de objetivos sociales y medioambientales, integrando la responsabilidad social como inversión estratégica en el núcleo de su estrategia empresarial, sus instrumentos de gestión y sus actividades.

Atendiendo a las consideraciones anteriores, los investigadores se orientaron a caracterizar el emprendimiento como proceso de responsabilidad social en instituciones de educación superior de América latina. Asumiendo el emprendimiento como un rasgo socio cultural que le permite a la persona definir su relación con el trabajo y la sociedad en general. Para el emprendedor el trabajo es una extensión de la propia persona y un medio de realización personal. Cabe destacar que esta investigación tiene como eje fundamental brindar condiciones socio cultural a los individuos y grupos, para que puedan desenvolverse de manera práctica y con altos niveles de integración social, orientándose a la búsqueda de niveles deseables y sostenibles de supervivencia y de procesos económicos sostenibles, con el objeto de mejorar su calidad de Vida. 


\section{ENFOQUE TEÓRICO SOBRE EMPRENDIMIENTO. CARACTERÍSTICAS GENERALES Y DEFINICIÓN.}

El proceso de emprendimiento ha ido experimentando un interés creciente en los últimos años debido a la iniciativa que ha generado desde diferentes áreas de la sociedad, sustentado la economía, gestión, sociología, psicología, política, convivencia social, entre otras. Dentro de este marco, la palabra emprendedor se orienta designar al empresario y emprendimiento, se refiere a las competencias, habilidades y destreza de los individuos para generar un proceso de negocio. Dentro de este marco, Krauss (2011), citado por Duran, Márceles \& Parra (2015) sostiene que el emprendedor es una persona que asume riesgos. Del mismo modo, una persona con mayor tolerancia al riesgo tendrá una mayor asunción de riesgo y, por lo tanto, un menor grado de aversión a este. Ello implicará que la persona tendrá menos miedo en auto-emplearse y en crear su propio emprendimiento.

Asimismo, Gutiérrez, (2006), asume que el emprendimiento es la capacidad de llevar a cabo proyectos de acción en el marco de un contexto de oportunidades identificadas y que contiene la suficiente energía para organizar los medios y recursos para aplicarlo. Es además un comportamiento individual y colectivo, el cual implica atributos y competencias básicas y algunas extraordinarias que viabilizan la posibilidad de la acción por emprender. Cabe destacar que, en Colombia, a partir de la Ley 1014 de 2006, se le dio vida a la Red Nacional para el Emprendimiento, la cual considera en su Artículo 1: El emprendimiento es una forma de pensar, razonar y actuar centrada en las oportunidades, planteada con visión global y llevada a cabo mediante un liderazgo equilibrado y la gestión de un riesgo calculado, su resultado es la creación de valor que beneficia a la empresa, la economía y la sociedad.

Para Duran, Márceles \& Parra (2015) en Colombia, el proceso de fomento de la cultura del emprendimiento implica la integración de las competencias básicas y ciudadanas, el emprendimiento y empresarismo al contexto institucional, lo cual se manifiesta al concebirla como el resultado de la construcción del proyecto educativo institucional y el proyecto de vida de sus estudiantes, donde su diseño e implementación contemple una mirada integral del ser humano.

Bajo este contexto, Suleiman (2015) indica que existen 5 características para ser un emprendedor exitoso:

Gráfico: 1. Características del emprendedor.

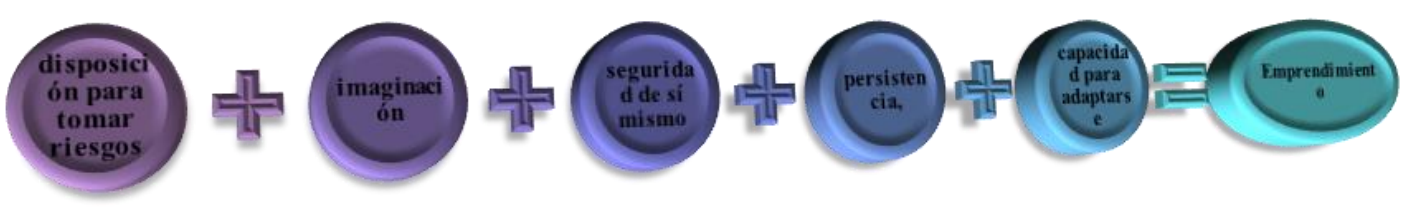

Fuente. Suleiman, (2015) 
A su vez en Emprende Pyme (2015) se indican doce características básicas: Pasión, visión, capacidad de aprendizaje, obtener resultados, determinación, creatividad e innovación, persistencia, sentido de oportunidad, trabajo en equipo, autoestima alta, asertividad y organización.

La vocación emprendedora y la generación de ideas para el Proyecto LATIN (2014) son ámbitos importantes de la creación de negocios, consideran en este estudio que una vez que el emprendedor ha tomado la decisión de iniciar un negocio, cumplida la etapa de Formación de la vocación emprendedora, entonces comienza la búsqueda de ideas que sean una real oportunidad de negocio. A continuación se incluye una breve descripción de cada tipo de emprendimiento:

\section{Cuadro 1 Tipología de emprendimiento}

Emprendimientos de base abierta

\begin{tabular}{|c|}
\hline \\
\hline $\begin{array}{c}\text { Base cultural y } \\
\text { creativa: }\end{array}$ \\
\hline $\begin{array}{c}\text { Emprendimientos } \\
\text { de base tecnológica } \\
\text { (EBT): }\end{array}$ \\
\hline $\begin{array}{c}\text { Emprendimientos } \\
\text { dinámicos }\end{array}$ \\
\hline $\begin{array}{c}\text { Emprendimientos } \\
\text { de base social }\end{array}$ \\
\hline $\begin{array}{c}\text { Emprendimientos } \\
\text { de base social: } \\
\text { Emprendimientos } \\
\text { inclusivos }\end{array}$ \\
\hline
\end{tabular}

Spin-off

Son emprendimientos de cualquier tipo y sector de la economía: agropecuario, industrial, comercial o de servicios, que por sus características no pueden incluirse en otras tipologías.

Dentro de esta categoría se fusiona creación, producción y comercialización de bienes y servicios basados en contenidos de carácter cultural Contiene actividades como: la edición impresa y multimedia, la producción cinematográfica y audiovisual, la artesanía y el diseño. Pueden incluirse también: la arquitectura, las artes plásticas, las artes del espectáculo, los deportes, la manufactura de instrumentos musicales, la publicidad y el turismo cultural. Incluyen productos tangibles (libros, cuadros, discos) y servicios artísticos, intelectuales o intangibles que tengan contenido creativo (un baile, una conferencia, una interpretación musical).

Son negocios generadores de valor, mediante la aplicación sistemática de conocimientos tecnológicos y científicos. Los EBT, generalmente operan en los sectores de alta tecnología, como: biotecnología, tecnologías de la información y las comunicaciones, química y nanotecnología, entre otros.

Son emprendimientos nuevos con alto potencial de crecimiento, que logran diferenciarse a través de un producto o servicio innovador, lo cual les permite en pocos años dejar de ser microempresas. En algunos contextos se incluye que tengan un horizonte exportador y que genere un gran número de empleos. (Kantis, Menéndez, \& Federico, 2012). Stuart-ups: emprendimiento de nueva creación generalmente asociado a emprendimientos de alto crecimiento y orientación tecnológica.

Son iniciativas emprendedoras que aplican estrategias de mercado, de creación de riqueza y empleo, para alcanzar un objetivo social. (Vernis \& Iglesias, 2010, )

incluyen a la población con algún grado de vulnerabilidad, o en la base de la pirámide, buscando ampliar el perfil de las personas o cambiar el statu quo para incorporarse a la sociedad, abriendo posibilidades a los grupos que sufren mayores dificultades de acceso al mercado de trabajo. ((Márquez \& Berger, 2009).

Constituyen uno de los tipos de empresas de base tecnológica cuya característica básica es que son empresas generadas a partir de resultados de investigación. Si se originan en una universidad son spin-off académicas y si se originan en un centro de investigación de una empresa son spin-off corporativas.

Fuente: Durán, Fuenmayor, Cárdenas \& Hernández (2016)

Se destaca que en el proyecto LATIN realizado en América latina en el año (2014) sugieren que las ideas son consecuencia de ciertos factores, dentro de los cuales pueden destacarse: la observación del entorno, la formación, la experiencia, los gustos y los deseos del emprendedor. En la mayoría de los 
emprendimientos, fundamentalmente en los que tienen un componente de innovación, es fundamental que el emprendedor haga una adecuada búsqueda de información, que le permitirá captar información del exterior que podrá analizar y utilizar para generar nuevas ideas.

Dentro de estas perspectivas, Duran (2016) manifiesta que es recomendable que las ideas tengan relación con la formación, la experiencia o el entorno del emprendedor. Una idea consiste en pensar las cosas diferentes, por ejemplo, considerar las ideas como solucionadores de problemas, también son una fuente de motivación en esta nueva era, los problemas medioambientales han dado origen a una serie de emprendedores y consumidores "verdes", con soluciones basadas en productos reciclados o fabricados con fibras naturales. Se perfila el emprendimiento desde un enfoque de desarrollo humano integral, el cual permite a la comunidad construir conocimientos y desarrollar hábitos, actitudes y valores necesarios para generar acciones emprendedoras, para la transformación del entorno y de la sociedad.

\section{1.- La responsabilidad social como compromiso y estrategia empresarial.}

La globalización, los tratados de libre comercio, el cambio climático han obligado a los gerentes y empresarios actuales a conducir las organizaciones no sólo por los caminos de la innovación y la competitividad con objetivos claros, sino que también los descalabros financieros de bancos, empresas y gobiernos de países desarrollados han provocado que sus representantes sean responsabilizados por prácticas antiéticas. Estas situaciones han llegado a llamar la atención de las escuelas de administración para desarrollar las habilidades que deben tener gerentes y líderes hoy día en el comportamiento ético más allá de adquirir formación y competencias en áreas como mercadeo, finanzas, economía y otras como ha sido tradicional en los programas de ciencias económicas, administrativas y contables y de algunos programas de ingeniería.

Hoy en países desarrollados los inversionistas de los mercados bursátiles buscan obtener información sobre los programas de RS de las empresas que elaboran, venden o importan un producto y/o servicio, dándole a estos programas un valor agregado con efectos sobre las cotizaciones de los títulos valores que se negocian en la bolsas de valores. Es a la rama del poder público a quien corresponde un papel fundamental en el tema de RS; al gobierno direccionar los recursos del gasto público a los segmentos de población que más lo requieran, utilizar la capacitación para hacer de los ciudadanos seres competitivos, la garantía de una adecuada asistencia en salud y una buena alimentación, el estímulo a la creación de trabajo productivo para disminuir los niveles de desigualdad y pobreza en la población más vulnerable.

Según Navarro, (2012), la responsabilidad social, "se basa en la mejora continua que permite a la empresa ser más competitiva, no a costa de, sino respetando y promoviendo el desarrollo pleno de las personas, de las comunidades en las que opera y del entorno". La responsabilidad la asumen de manera voluntaria cuando sus acciones afectan con impactos el medio ambiente, y las condiciones de desarrollo 
humano. En este orden de ideas, los investigadores han tomado la oportunidad para resaltar un modelo de RS de un gran empresario que dejó un valioso legado social, se trata de Manuel Carvajal Sinesterra fundador de la empresa que lleva su apellido, que es en estos tiempos una de las principales multinacionales colombianas con presencia en 50 países.

Ese gran mérito es la Fundación Carvajal creada en 1961 y que posee el 40\% de las acciones de la firma Carvajal S.A. que se creó para conseguir una fuente de financiación de recursos para invertirlos en vis, a la capacitación y a la salud. En materia de vivienda se han beneficiado más de 300 mil personas, en capacitación se han entrenado 200 mil personas de diversas edades y en salud se logró reducir la tasa de mortalidad infantil en cerca del $90 \%$, además de otras inversiones en cultura y recreación para una población de muy escasos recursos.

\section{2.- Responsabilidad social de la universidad para el desarrollo sostenible de la región a través del emprendimiento.}

La universidad es fundamento y condición del desarrollo no solo profesional y personal sino social de la región. Ante la carencia de oportunidades laborales muy pocas universidades en Colombia, algunas si lo hacen, es construir un marco de responsabilidad bajo la óptica de fomentar en alianza con las entidades del sector público que se relacionan con estos temas y los empresarios locales, una cultura emprendedora para generar nuevos proyectos no importa en principio su tamaño. En casi todas las escuelas de negocios en Colombia en unas mejores que en otras, han formado generaciones académicamente y lo siguen haciendo, para ser empleados y no emprendedores.

Se trata de estimular la capacidad de crear y ofrecer en el mercado bienes y servicios bien sea mejorados o completamente nuevos o distintos a los ya existentes. En casi todos los países del mundo donde es permitido desarrollar iniciativas privadas de negocio, observamos que las más grandes empresas han sido creadas por emprendedores a través de empresas de familia donde ha existido un ingrediente obligatorio para el crecimiento como es la innovación y relacionando la iniciativa de emprender una idea de negocio con la cultura, formación y modelos familiares.

Es por esto que en opinión de expertos tales como Duran et al (2015) la formación de un emprendedor debe empezar en el ambiente y entorno familiar, luego en el colegio y posteriormente en la universidad. Los programas de gobierno en asocio con los programas académicos podrían forjar una nueva y dinámica generación de empresarios que mejoren las condiciones de vida e indicadores sociales de la población logrando un mejor bienestar para el país.

Además para todos los programas académicos según Paz, Prieto y García (2016) se puede estimular el espíritu emprendedor que no es un tema exclusivo para los programas de administración, ingeniería o 
economía. Existe un problema conceptual que tiene un origen cultural en los habitantes de la región Caribe y es el de creer que ser empleado garantiza una estabilidad económica de por vida, pero eso no es cierto. El ahorro para la pensión no genera riqueza para un país, la inflación y los periodos de cambios económicos no garantizan la estabilidad, a esto hay que agregarle la esperanza de vida que ya alcanza los 85 años. Siendo empresario también se puede cotizar para lograr la pensión, incluso a una edad relativamente temprana a través de los fondos de pensiones privadas.

Es así como los estudiantes de todos los programas deben entender desde los primeros semestres la diferencia entre ser empleado y ser empresario para generar riqueza, nuevos proyectos, mayores ingresos, estatus profesional, reconocimiento social y felicidad de ser dueño de un negocio y no depender de un empleo para subsistir. Dentro de los temas de una agenda para la realización de un plan de actividades que contribuyan a mejorar la competitividad de la región en el campo académico, se sugiere capacitar a los estudiantes en identificar las oportunidades de negocio, en la aceptación al cambio, en el deseo de auto realización, en la conformación de equipos empresariales, inculcar valores como la solidaridad, la rectitud, la responsabilidad social, la constancia y la perseverancia buscando un cambio de costumbres y creencias para crear ciudadanos adaptados a un mundo cada vez más integrado en las esferas económica, cultural y política.

Por último debe haber un compromiso de la sostenibilidad en ser socialmente responsables, si entendemos este concepto como útil e importante en nuestras vidas que nos permitan apropiarnos de los objetivos que la identifican para lograr su construcción, desarrollo y mantenimiento. La RS debe convertirse en una cultura social que quede inmersa en los procesos educativos, donde los proyectos pueden ser desarrollados en participación estrecha con la empresa privada, con los departamentos y municipios de la región.

De acuerdo a lo planteado, Martínez (2013), alega que las empresas no funcionan de manera aislada con respecto a la sociedad que los rodea. De hecho, su capacidad para competir depende de los lugares en que opera. La mejora de educación, suele considerarse una cuestión social, pero el nivel de formación de la fuerza de trabajo local afecta sustancialmente el nivel competitividad potencial de una empresa. Cuanta más relación con el negocio de una empresa tiene una mejora social, más conduce también a generar beneficios económicos. Una empresa que tenga posibilidades de ayudar al mejoramiento de la capacitación de los habitantes de su entorno, mejora su contexto competitivo.

En este sentido, las instituciones de educación superior han tomado como obligación incorporar principios que implican a los grupos de interés afectados por sus acciones, entre ellos la creación de valor, voluntariedad, adaptación e integración a los procesos sociales, ello promueve la eficiencia y eficacia de las mismas al adaptarse a la realidad social en la que actúa, tomando en cuenta el entorno, se presupone 
además la capacidad de tener suficiente transparencia con los grupos de interés, internos y externos, con el fin de dar credibilidad y rigor a la RS institucional.

Grafico 2. Principios de la responsabilidad social en IES.

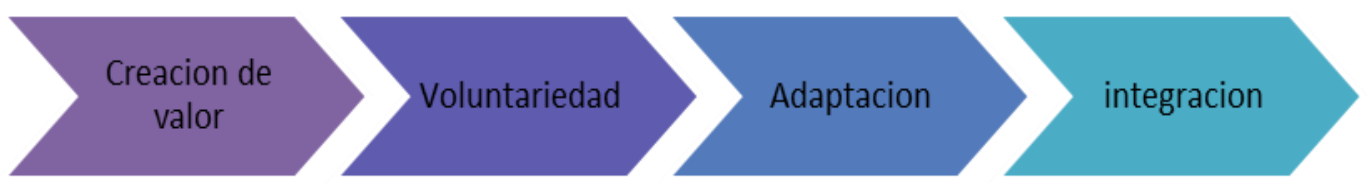

Fuente: Durán, Fuenmayor, Cárdenas \& Hernández (2016)

\section{MÉTODO}

\section{$\checkmark$ Diseño}

El presente estudio se sustenta en el paradigma positivista, además de tipo descriptivo aplicado enmarcado con un diseño no experimental de campo, pues su objetivo ha sido la evaluación del emprendimiento como proceso de responsabilidad social en instituciones de educación superior en Colombia y Venezuela. Cabe destacar que en el momento de aplicar el instrumento de recolección de datos, especificó las propiedades importantes de personas, grupos, comunidades o cualquier otro fenómeno al cual fue sometido el análisis.

También se considera de campo, porque el mismo realizó propiamente en el lugar donde se suscitaron los hechos, en las instalaciones de la Instituciones de educación superior seleccionadas, dado que el diseño de la investigación, es no experimental, transversal, coincide con el hecho de que las variables no han sido manipuladas, ni modificadas por los investigadores, y la recolección de datos se hizo en un único momento, obteniendo de esta forma una sola medición.

\section{$\checkmark$ Participantes}

La población estuvo conformada por 50 Docentes relacionados con el proceso de emprendimiento de Colombia, y Venezuela. Cuyas características se presentan en el Cuadro 2. Esta población fue analizada en su totalidad mediante un censo poblacional, por lo cual no se utilizó la técnica del muestreo para dicho estudio.

\section{Cuadro 2. Población}

\begin{tabular}{|c|c|c|}
\hline $\begin{array}{l}\text { Número de } \\
\text { personas }\end{array}$ & País & Instituciones \\
\hline 25 & Colombia & $\begin{array}{c}\text { Corporación universitaria Latinoamericana, } \\
\text { Universidad de la Costa }\end{array}$ \\
\hline 25 & Venezuela. & $\begin{array}{l}\text { Universidad Rafael Belloso Chacín- Venezuela } \\
\text { Universidad Rafael María Baralt- Venezuela }\end{array}$ \\
\hline 50 & & \\
\hline
\end{tabular}




\section{$\checkmark$ Técnicas}

Con respecto la técnica de recolección de datos utilizada en esta investigación, es la observación por encuesta, para la obtención de datos, se seleccionó el cuestionario como instrumento de investigación a utilizar. Ambos constituidos por quince ítems (15), los cuales contienen cinco opciones para que cada uno de los sujetos encuestados seleccionara la opción pertinente, para efectos de esta investigación, la escala de opciones viene dada por las siguientes alternativas. Siempre, Casi siempre, a veces, casi nunca y Nunca

\section{RESULTADOS}

En el punto siguiente se presenta el análisis de los resultados alcanzados luego de la aplicación del cuestionario a la población objeto de estudio, para ello se consideró pertinente reflejarlo por datos agrupados, en función de la variable, dimensión e indicador. Bajo este contexto, se sustentan con estadística descriptiva, procesadas a partir del baremo presentado consecutivamente, este se construyó sobre la base de las puntuaciones utilizadas en la escala, la cual va de 1 a 5.

Ubicándose en los rangos muy bajos (1- 1.79), bajo (1.80 - 2.59), medio (2.60 - 3.39), alta (3.40- 4.19) y muy alta $(4.20-5)$.

Tabla 1. Características del emprendimiento

\begin{tabular}{|c|c|c|c|c|c|c|c|c|c|c|c|c|c|c|}
\hline \multirow[t]{3}{*}{ Indicador } & \multirow[t]{3}{*}{ Items } & \multicolumn{10}{|c|}{ Alternativas de Respuestas } & & & \\
\hline & & \multicolumn{2}{|c|}{ Siempre } & \multicolumn{2}{|c|}{ Casi siempre } & \multicolumn{2}{|c|}{ A veces } & \multicolumn{2}{|c|}{ Casi nunca } & \multicolumn{2}{|c|}{ Nunca } & \multicolumn{3}{|c|}{ Promedio } \\
\hline & & FA & FR & FA & FR & FA & FR & FA & FR & FA & FR & ítem & indic & dmens \\
\hline \multirow{3}{*}{$\begin{array}{l}\text { Disposición para } \\
\text { tomar riesgos }\end{array}$} & 1 & 37 & $74 \%$ & 7 & $14 \%$ & 6 & $12 \%$ & 0 & $0 \%$ & 0 & $0 \%$ & 4,5 & \multirow{3}{*}{4,5} & \multirow{15}{*}{3,5} \\
\hline & 2 & 42 & $84 \%$ & 6 & $12 \%$ & 2 & $4 \%$ & 0 & $0 \%$ & 0 & $0 \%$ & 4,7 & & \\
\hline & 3 & 27 & $54 \%$ & 13 & $26 \%$ & 10 & $20 \%$ & 0 & $0 \%$ & 0 & $0 \%$ & 4.0 & & \\
\hline \multirow{3}{*}{$\begin{array}{l}\text { Capacidad para } \\
\text { adaptarse }\end{array}$} & 4 & 29 & $58 \%$ & 9 & $18 \%$ & 12 & $24 \%$ & 0 & $0 \%$ & 0 & $0 \%$ & 2.90 & \multirow[t]{3}{*}{4} & \\
\hline & 5 & 35 & $70 \%$ & 14 & $28 \%$ & 1 & $2 \%$ & 0 & $0 \%$ & 0 & $0 \%$ & 4.58 & & \\
\hline & 6 & 34 & $68 \%$ & 9 & $18 \%$ & 7 & $14 \%$ & 0 & $0 \%$ & 0 & $0 \%$ & 4.44 & & \\
\hline \multirow[t]{3}{*}{ Persistencia } & 7 & 14 & $28 \%$ & 15 & $30 \%$ & 16 & $32 \%$ & 6 & $12 \%$ & 0 & $0 \%$ & 3.74 & \multirow[b]{3}{*}{3,5} & \\
\hline & 8 & 19 & $38 \%$ & 17 & $34 \%$ & 16 & $32 \%$ & 0 & $0 \%$ & 0 & $0 \%$ & 3,48 & & \\
\hline & 9 & 11 & $22 \%$ & 16 & $32 \%$ & 16 & $32 \%$ & 3 & $6 \%$ & 0 & $0 \%$ & 3,36 & & \\
\hline \multirow{3}{*}{$\begin{array}{l}\text { Seguridad de sí } \\
\text { mismo }\end{array}$} & 10 & 4 & $8 \%$ & 18 & $36 \%$ & 17 & $34 \%$ & 11 & $22 \%$ & 0 & $0 \%$ & 3,20 & \multirow[t]{3}{*}{3} & \\
\hline & 11 & 7 & $14 \%$ & 22 & $44 \%$ & 21 & $42 \%$ & 0 & $0 \%$ & 0 & $0 \%$ & 2,24 & & \\
\hline & 12 & 15 & $30 \%$ & 22 & $44 \%$ & 13 & $26 \%$ & 0 & $0 \%$ & 0 & $0 \%$ & 3,46 & & \\
\hline \multirow[t]{4}{*}{ Imaginación } & 13 & 25 & $50 \%$ & 18 & $36 \%$ & 7 & $14 \%$ & 0 & $0 \%$ & 0 & $0 \%$ & 4,32 & \multirow[t]{4}{*}{4,1} & \\
\hline & 14 & 29 & $58 \%$ & 19 & $38 \%$ & 2 & $4 \%$ & 0 & $0 \%$ & 0 & $0 \%$ & 4,46 & & \\
\hline & 15 & 14 & $28 \%$ & 14 & $28 \%$ & 13 & $26 \%$ & 9 & $18 \%$ & 0 & $0 \%$ & 3,54 & & \\
\hline & & & & & & & & & & & & & & \\
\hline
\end{tabular}

Fuente: Durán, Fuenmayor, Cárdenas \& Hernández (2016)

En la tabla 1 se muestran los resultados correspondientes a las Características del emprendimiento lo cual da respuesta al primer objetivo específico de esta investigación, se consideraron cinco indicadores. 
Con respecto a la dimensión su promedio se ubicó en 3,5 generando una alta tendencia en el emprendimiento. Con respecto a los indicadores se describen cada uno por separado. Con respecto a la Disposición Su promedio se ubicó en 4,5 ello indica que la población considera que esta dimensión tiene una ata tendencia en el emprendimiento. Se observa en la tabla las tendencias de las respuestas según resultados en la categoría Siempre de $74 \%, 84 \%$ 54\%, mientras un 14\%, $12 \%$ y $26 \%$ consideraron la opción casi siempre.

Por otra parte la Capacidad para adaptarse, alcanzó un promedio de 4, con unos resultados de 58\%, 70\% y $68 \%$ en la opción siempre, además $18 \%, 28 \%$, 18\% en casi siempre, mientras que en la opción a veces el $24 \%, 2 \%$ y $7 \%$. En cuanto al indicador Persistencia, su promedio arrojo un resultado de 3,5 forjando un alto nivel en emprendimiento, bajo este contexto, los resultados fueron los siguientes, en la opción siempre se ubicó el 28\%, 38\% y 22\%, además en la opción casi siempre el 30\% 34\% y 32\%, datos que permiten confirmar que la mayoría de la población encuestada considera que existe un alto nivel en emprendimiento, por otra parte en la alternativa a veces la tendencia fue del $32 \%$, mientras que para casi nunca esta solo el 12 y $6 \%$.

Seguidamente el indicador seguridad en sí mismo generó un promedio de 3, indicando que el nivel de seguridad que tiene el cliente respecto al servicio es medio, en ese sentido las mayores tendencias se ubicaron en casi siempre y a veces, tales como $36 \%, 44 \%$ y $44 \%$ para casi siempre, por otra parte en la alternativa a veces el 34\%, $42 \%$ y 26\%, mientras para la opción casi nunca hubo un 22\% para el items 10 . No obstante solo el 4\%, 7\% y 15\% consideraron siempre. En el indicador imaginación considera que las tendencias están hacia un alto nivel, puesto que su promedio es 4,1 en función del baremo, sus resultados son el $50 \%, 58 \%$ y $28 \%$ para la opción siempre aunado al 36\%, 38\% y $28 \%$ para casi siempre, Con respecto a la alternativa a veces contestaron el $14 \%, 4 \%$ así como el $26 \%$, en tanto en tanto sol el $18 \%$ consideró casi nunca para el ítem 15.

Tabla 2. Principios de la Responsabilidad Social

\begin{tabular}{|c|c|c|c|c|c|c|c|c|c|c|c|c|c|c|}
\hline \multirow[t]{3}{*}{ Indicador } & \multirow[t]{3}{*}{ Items } & \multicolumn{10}{|c|}{ Alternativas de Respuestas } & & & \\
\hline & & \multicolumn{2}{|c|}{ Siempre } & \multicolumn{2}{|c|}{ Casi siempre } & \multicolumn{2}{|c|}{ A veces } & \multicolumn{2}{|c|}{ Casi nunca } & \multicolumn{2}{|c|}{ Nunca } & \multicolumn{3}{|c|}{ Promedio } \\
\hline & & FA & FR & FA & FR & FA & FR & FA & FR & FA & FR & ítem & indic & dmens \\
\hline \multirow[t]{3}{*}{ Creación de valor } & 16 & 9 & $18 \%$ & 6 & $14 \%$ & 10 & $20 \%$ & 21 & $42 \%$ & 4 & $8 \%$ & 4.2 & \multirow[t]{3}{*}{3,49} & \multirow{9}{*}{3,34} \\
\hline & 17 & 2 & $4 \%$ & 15 & $30 \%$ & 11 & $22 \%$ & 18 & $36 \%$ & 4 & $8 \%$ & 3 & & \\
\hline & 18 & 3 & $6 \%$ & 8 & $16 \%$ & 33 & $66 \%$ & 2 & $4 \%$ & 4 & $8 \%$ & 3.2 & & \\
\hline \multirow[t]{3}{*}{ Voluntariedad } & 19 & 2 & $4 \%$ & 4 & $8 \%$ & 4 & $8 \%$ & 11 & $22 \%$ & 30 & $60 \%$ & 2.9 & \multirow[t]{3}{*}{2,94} & \\
\hline & 20 & 2 & $4 \%$ & 5 & $10 \%$ & 7 & $14 \%$ & 30 & $60 \%$ & 6 & $12 \%$ & 2.9 & & \\
\hline & 21 & 22 & $44 \%$ & 12 & $24 \%$ & 3 & $6 \%$ & 9 & $18 \%$ & 4 & $8 \%$ & 3.1 & & \\
\hline \multirow[t]{3}{*}{ Adaptación } & 22 & 2 & $4 \%$ & 4 & $8 \%$ & 4 & $8 \%$ & 11 & $22 \%$ & 30 & $60 \%$ & 1.8 & \multirow[b]{3}{*}{2.38} & \\
\hline & 23 & 2 & $4 \%$ & 5 & $10 \%$ & 7 & $14 \%$ & 30 & $60 \%$ & 6 & $12 \%$ & 2.5 & & \\
\hline & 24 & 9 & $18 \%$ & 4 & $8 \%$ & 22 & $44 \%$ & 12 & $24 \%$ & 3 & $6 \%$ & 3.2 & & \\
\hline
\end{tabular}




\begin{tabular}{|c|c|c|c|c|c|c|c|c|c|c|c|c|c|}
\hline integración & $\mathbf{2 5}$ & 44 & $88 \%$ & 1 & $2 \%$ & 3 & $6 \%$ & 2 & $4 \%$ & 0 & $0 \%$ & $\mathbf{4 . 8}$ & $\mathbf{4 . 5}$ \\
\cline { 2 - 33 } & $\mathbf{2 6}$ & 44 & $88 \%$ & 1 & $2 \%$ & 3 & $6 \%$ & 2 & $4 \%$ & 0 & $0 \%$ & $\mathbf{4 . 8}$ \\
\cline { 2 - 15 } & $\mathbf{2 7}$ & 43 & $86 \%$ & 5 & $10 \%$ & 2 & $4 \%$ & 0 & $0 \%$ & 0 & $0 \%$ & $\mathbf{4 . 4}$ \\
\hline
\end{tabular}

Fuente: Durán, Fuenmayor, Cárdenas \& Hernández (2016)

La tabla 2, muestra los resultados asociados a los principios de la responsabilidad social en las instituciones de educación superior, bajo este contexto, los resultados indican la tendencia hacia el nivel medio con un promedio de 3,34. En este sentido los porcentajes presentados en la tabla indican que el indicador Creación de valor, hacia aspecto de insatisfacción, cuando se le pegunto a la población si se generan procesos de creación de valor por parte de las IES, muestra un porcentaje del $42 \%$ quienes consideraron la opción casi nunca, no obstante un $20 \%$ asumió la alternativa a veces, un $18 \%$ manifestó que siempre mientras un $14 \%$ casi siempre, así como un $4 \%$ nunca.

Por otro lado el ítem referido al sustento de una idea de negocios, se obtuvo en la opción en casi nunca un $36 \%$ de la población, además un $22 \%$ considero a veces. Asimismo se les pregunto si Involucra los aspectos organizacionales en la atención al entorno, un 66\% de la población manifestó a veces, mientras un $16 \%$ considera casi siempre. Bajo este contexto, se puede inferir que el personal considera que no existe vinculación con la creación de valor hacia los grupos de interés, generando un promedio de 3,49

Con respecto al indicador Voluntariedad, está orientado en primer término a un nivel medio de responsabilidad social, en virtud de que tiene un promedio en 2,94. Con respecto a las tendencias el $60 \%$ manifestó que nunca se generan actividades en forma voluntaria para promover emprendimiento, un $22 \%$ que casi nunca. Asimismo el $60 \%$ considero la opción casi nunca con respecto a si las IES, les ofrece capacitación para el apoyo a emprendedores, mientras un $14 \%$ a veces y un $12 \%$ nunca.

En cuanto al indicador adaptación, se ha generado una posición diversa con respecto al ítem no el $60 \%$ consideró que en las IES, no existen políticas que le proporcionen desarrollo con respecto a cómo proponer proyectos de emprendimiento, el personal respondió la opción nunca, asimismo un $22 \%$ casi nuca. Por otra parte un $60 \%$ afirmo que cuando el docente busca involucrarse con las actividades de emprendimiento logra las metas, casi nunca se les reconoce el trabajo, apoyado por un $14 \%$ quienes consideran que solo a veces y un $12 \%$ opto por la opción nunca. Además el $44 \%$ manifestó que a veces, suelen evaluar estas actividades como parte del desempeño para promoverlo, a su vez un $24 \%$ casi nunca mientras un $18 \%$ casi siempre. El promedio del indicador fue de 2,38 indicando en este aspecto un nivel bajo de responsabilidad social.

Asimismo el indicador integración, presenta una alta tendencia hacia la responsabilidad social, dado que la mayoría de la población se orientó a considerar la opción siempre con el 88 \% para el ítem 25 y 26 , 
mientras que para el 27 fue el 86\%. Cuando se les pregunto si en las IES, le da oportunidad de usar al máximo sus habilidades, además la estructura de las operaciones laborales involucra procesos gerenciales para solucionar los problemas generados en la organización, por otra parte si emplean herramientas estratégicas para estructurar las operaciones en función de promover el emprendimiento. Su promedio alcanzo un puntaje de 4,5, referido a un nivel muy alto con respecto al baremo de interpretación

\section{DISCUSIÓN}

Como se observó en los resultados los factores críticos en el comportamiento emprendedor se relacionan con las siguientes variables (Acs, 2007; Wennekers, 2006): El nivel de educación. La posibilidad de que los individuos participen en iniciativas de emprendimiento innovador está ligada al logro de niveles elevados de educación. Asimismo el desarrollo de habilidades. La educación formal debe estar complementada por el logro de habilidades para el emprendimiento. Estas habilidades, definidas como la capacidad para hacer, están relacionadas con los niveles de educación, pero se adquieren fundamentalmente a partir de la experiencia. En otro sentido, se genera la capacidad para la identificación de oportunidades. Esta es la capacidad para articular un orden a partir del caos del entorno, descubrir necesidades insatisfechas. En los consumidores y crear soluciones para esas necesidades (Wennekers, 2006). Asimismo, se percibe la preferencia individual por el riesgo. Los emprendedores se caracterizan por tener alta disposición individual a la toma de riesgos, pues están dispuestos a aceptarlo en situaciones donde consideran que pueden obtener amplias ganancias.

Con respecto a los Recursos sicológicos (psychological endowments), las características sicológicas de los individuos determinan la capacidad individual para el emprendimiento. Ellas incluyen la creatividad, la perseverancia, el control interno, la iniciativa, la autonomía, la motivación por el logro, la disposición a abrirse a experiencias diversas y la capacidad de incorporar las lecciones de la experiencia en las decisiones, entre otras.

Dentro de este marco, a la Responsabilidad Social, se obtuvo que hay una respuesta efectiva, ya que existe equidad en tanto que permite la expansión de sus beneficios sin ninguna discriminación, comprende el acceso de oportunidades para un desempeño exitoso y proporcionan mejores logros educativos, asimismo se pudo constatar el bajo nivel de existencia de la creación de valor, de la voluntariedad por parte del perenal para promover el emprendimiento, puesto que en las universidades surgen nuevos fenómenos, por ende la integración como principio genera un nivel alto de existencia que a su vez producen respuestas acordes a las necesidades del entorno.

En virtud de ello, Martínez (2013), sostiene que la mejora de educación, suele considerarse una cuestión social, pero el nivel de formación de la fuerza de trabajo local afecta sustancialmente el nivel 
competitividad potencial de una empresa. Cuanta más relación con el negocio de una empresa tiene una mejora social, más conduce también a generar beneficios económicos

También se puede definir la RS, como la suma de esfuerzos y voluntades, individuales y colectivas, públicas y privadas, en procura de obtener mejores niveles de vida para la sociedad, se convierte en un requisito indispensable para la obtención del título académico correspondiente y en consecuencia para el ejercicio profesional.

Atendiendo a las consideraciones anteriores la Responsabilidad social de las Universidades conducen al planteamiento de que, en virtud de comprender el surgimiento de los nuevos fenómenos, las Universidades producen respuestas acordes a sus necesidades, igualmente, La universidad contempla la participación de los profesores en el estudio, comprensión y alternativas de solución de problemas sociales de la realidad nacional y que el docente ofrece a los estudiantes las orientaciones necesarias, para ejecutar procesos de emprendimiento.

Seguidamente tomando en consideración los planteamientos antes mencionados, y analizando la responsabilidad social de las instituciones de educación superior, la población ha considerado que el emprendimiento, es parte del cumplimiento de la responsabilidad social de las Universidades.

Luego de realizar el estudio y caracterizar el emprendimiento como proceso de responsabilidad social en las instituciones de educación superior, se ha llegado a la conclusión estudios indicarían que se podría acelerar la transición hacia el emprendimiento innovador logrando fortalecer las conexiones entre los actores sociales, en torno a la innovación y la transferencia de conocimiento. Dado que existe un alto potencial de personas emprendedoras.

También se pudo comprobar la materialización de actitudes emprendedoras de los ciudadanos en el desarrollo de actividades y proyectos orientados a la creación de empresa o unidades de negocio con perspectiva de desarrollo sostenible. Dado que se han desarrollado competencias y habilidades emprendedoras en el ciudadano para trabajar en equipo, así como afrontar situaciones diversas que se dan en la convivencia ciudadana o en la vida cotidiana.

Además existe desde las universidades una vinculación orientada a desarrollar capacidades de autonomía personal, de juicio y de responsabilidad personal, buscando la comprensión y aceptación en las relaciones interpersonales y de interdependencia; habilidad en la resolución de conflictos y visión para afrontar proyectos comunes, buscando la ppromoción de la Asociatividad Emprendimiento y fortalecimiento de la generación de ingresos, desde las instituciones de educación superior sustentados en el cumplimiento de su responsabilidad social.

\section{6.- REFERENCIAS}


Acción Empresarial. (2003). El ABC de la RSE en Chile y en el mundo. Santiago de Chile: Acción Empresarial.

Areque, R., \& Montero, J. (2006). La Responsabilidad Social de la Empresa a Debate. Barcelona, España: Icaria.Center for Advancement of Social Entrepreurship (CASE). (s.f.). What is Social Entrepreneurship? Recuperado el 27 de Noviembre de 2015, de http://www.caseatduke.org/about/sedefinition.htmL

Certo, S. (2007). Administración Moderna. Prentice Hall. Colombia. Octava Edición.

Cachón C. (2001). La Empresa Social: la Responsabilidad Social de la Empresa. España. Editorial Ariel.

Drucker, P. (1985). Innovation and entrepreneurship. New York: Harper and Row

Duran Sonia, Márceles Víctor y Parra Margel (2015) Potenciación de habilidades para el desarrollo de emprendedores exitosos en el contexto universitario. Revista Opción, Año 31, No. 77 (2015): 200 215 ISSN 1012-1587. Universidad Del Zulia. Maracaibo, Venezuela.

Durán Sonia. (2016) "La formación del emprendimiento un elemento en el contexto internacional" En: ed: Corporación Universitaria Latinoamericana. Barranquilla Colombia.

Duran Sonia Ethel, Parra Fernández Margel Alejandra, Martínez ventura, Jairo Luis Hernández Palma, Hugo Gaspar, Silvera Sarmiento asteria de Jesús Alvarino Cruz, Cesar (2016) "El desarrollo tecnológico e innovación: elementos esenciales de emprendimiento" ed: Corporación Universitaria Latinoamericana. Barranquilla Colombia.

Emprende Pyme (2015) Doce características básicas de los emprendedores de éxito. Extraído el día 6 de Abril del 2016 desde: http://www.emprendepyme.net/12- caracteristicas-basicas-de-losemprendedores-de-exito.html

Gutiérrez, Guillermo. 2009. "Habilidades del emprendedor y características emprendedoras personales". Guía fácil para emprendedores. Lecc-6. Ed. Aula Fácil. Bogotá (Colombia). Disponible en: http://www.aulafacil. com/emprendedores-guía-fácil/curso/Lecc-6.htm Fecha de consulta: 16 de Febrero de 2016.

Jaramillo Leonardo (2008). "Emprendimiento: Concepto básico en competencias". Revista Lumen. Edición número 7. Ed. Instituto de educación IESE. Universidad del Norte. Barranquilla (Colombia).

Kantis, H. (2007) Propuesta de Programa de Emprendimiento Juvenil para Chile. Consejo Nacional de Equidad y trabajo de Chile. Santiago de Chile 
Krauss Catherine. (2011). "Actitudes emprendedoras de los estudiantes universitarios: El caso de la Universidad Católica del Uruguay”. Revista Científica Dimensión Empresarial. Vol. 9 No. 1, Enero-Junio: págs. 28- 40. Ed. Universidad Católica del Uruguay. Montevideo (Uruguay).

Koontz, H. O’Donnell, Weihrich. (2007). Elementos de la Administración. McGraw Hill. Tercera Edición. México.

López, J. (2012). Modelos actitudinales y emprendimiento sostenible. Cuaderno Interdisciplinar de Desarrollo Sostenible | ISSN 1889-0660. Universidad de Almería. Abril 2012. España.

Martínez, H. (2010) Responsabilidad Social y Ética Empresarial. Primera Edición. ECOE, Colombia.

Navarro García, Fernando (2012) Responsabilidad Social Corporativa Teoría y práctica. ESIC editorial.

Parra, M., \& Durán, S. (2014). Desarrollo organizacional y estrategias de tecnoformación en instituciones de educación superior colombianas. Dictamen Libre, 14(14/15), 14-23.

Paz, A., Prieto, R. \& García, J. (2016).responsabilidad social como alianza para el desarrollo sostenible en empresas carboníferas. Libro: Energías Renovables y Redes Global de Conocimiento. Aportes de investigaciones. Servicio Nacional de Aprendizaje - SENA. Regional Guajira. P.p. 216-231.

Perdomo, Y., \& Prieto, R. (2010). El liderazgo como herramienta de competitividad para la gerencia del servicio. CICAG, 6(2), 19-31.

Prieto, R., \& Paz, A. (2011). Responsabilidad social universitaria. Una visión estratégica de corresponsabilidad EN LA UNIVERSIDAD DR. JOSÉ GREGORIO HERNANDEZ. Maracaibo Venezuela.

Proyecto Latin-America Latina (2014) en http://proeva.edu.uy/institucional/proyectos/latin/ Recuperado enero 2016.

Tan, W. L. (2005). Defining the 'Social' in 'Social Entrepreneurship': Altruism and Entrepreneurship. International Entrepreneurship and Management Journal, 353-365.

TAN, W. L. (2007). Entrepreneurship as a wealth creation and value-adding process. Journal of Enterprising Culture, 15(2), 101-105.

Timmons, J., \& Spinelli, S. (2004). New venture strategies: Entrepreneurship for the 21st century. Burr Ridge,

IL:

Irwin-McGraw-Hill

Publishers. 